\title{
The Optimization of Airport Management Based on Collaborative Optimization of Flights and Taxis
}

\author{
Xiaobing Ding $\mathbb{D},{ }^{1,2}$ Zhigang Liu $\mathbb{D}^{2},{ }^{2}$ Gan Shi, ${ }^{2}$ Hua Hu, ${ }^{2}$ Jiaping Chen, ${ }^{2}$ Kaihe Yang, \\ Su Wan, ${ }^{2}$ and Jinlong $W u^{3}$ \\ ${ }^{1}$ School of Naval Architecture, Ocean and Civil Engineering, Shanghai Jiao Tong University, Shanghai 200240, China \\ ${ }^{2}$ School of Urban Railway Transportation, Shanghai University of Engineering Science, Shanghai 210620, China \\ ${ }^{3}$ School of Transportation Engineering, Yangzhou Polytechnic Institute, Yangzhou 225100, China
}

Correspondence should be addressed to Zhigang Liu; lzg@sues.edu.cn

Received 10 November 2021; Revised 14 December 2021; Accepted 12 January 2022; Published 4 February 2022

Academic Editor: Zeshui Xu

Copyright $(92022$ Xiaobing Ding et al. This is an open access article distributed under the Creative Commons Attribution License, which permits unrestricted use, distribution, and reproduction in any medium, provided the original work is properly cited.

How to efficiently guide passengers and ensure the order of airport operation is an urgent transport problem for airport management. Based on the analysis of the factors that affect the driver's decision-making, this paper deeply explores the collaborative association of the core factors, such as the number of flight arrivals in different periods and the average seeking distance of taxis. Firstly, according to the GPS data of taxis, the paper uses clustering algorithm to get the average passenger-seeking time from the airport and makes matching interaction between the number of flights based on time distribution and the average passenger-carrying capacity of vehicles in the parking garage, so as to build a decision-making model based on the number of taxis $\mathrm{N}$; secondly, it takes passenger safety and traffic order as the priority and uses $\mathrm{M} / \mathrm{M} / \mathrm{S}$ queuing model to integrate the two factors. Taking the maintenance cost and passenger evacuation time as constraints, the judgment condition of minimum cost $Z_{\min }$ and the optimal number of boarding points $S_{m}$ are solved. Finally, taking the flight and taxi data of Shanghai Hongqiao Airport as an example, the driver's decision-making standard is simulated, and the accuracy of the model is verified by the deviation rate. It can provide decision-making support for taxi management of urban transportation hub and rapid evacuation of airport passengers, so as to realize the collaborative optimization of airport flight arrival and taxi carrying order.

\section{Introduction}

When arriving at the airport, taking taxi is one of the main choices of passengers. Most of the airports in China separate the drop off (departure) and pick up (arrival) channels. Taxi drivers who drop off passengers to the airport will face two choices: (1) Proceed to the arrival area to wait for carrying passengers back to the city. Taxis must wait in a line at the designated "taxi storage pool" and enter the venue according to the "first come, first served" queue. The waiting time depends on the number of taxis and passengers in the queue, and a certain time cost is required. (2) Return directly to the city to search passengers. Taxi drivers will pay no-load fees and may lose potential passenger revenue. Generally, the driver can make a choice based on the number of flights arriving at the current time and the number of taxis already in the storage pool, combined with the experience. If passengers want to take a taxi after getting off the plane, they must line up at the designated boarding area and queue up in order. Airport taxi management personnel are responsible for letting taxis into the "riding area" in "quantitative batches" and arranging a certain number of passengers to board the bus. In actual operations, there are still many factors that affect the determination and uncertainty of taxi drivers' decision-making. Their correlations are different, and their effects are also different.

At the airport, there are often situations in which taxis should queue up to wait for passengers and passengers also should line up for boarding the taxi. How to set the boarding point of passengers to ensure the safety and order of passengers should be determined so that the overall efficiency of the operation can be the highest. The taxi driver's income is 
related to the distance of passengers. Taxi drivers cannot refuse to carry passengers, no matter whether the passengers' destination is far or near, but the taxi is allowed to carry passenger multiple times. How can the plan be arranged to make the income of the taxi as balanced as possible? All of the above issues require in-depth study of the influence mechanism of relevant factors in taxi driver's decisionmaking, comprehensively considering the changing law of the number of airport passengers and the income of taxi drivers, establishing a taxi driver selection decision-making model, and giving drivers' corresponding strategies.

Airport taxi carrying optimization problem is a typical decision-making problem. At present, the mainstream decision-making theories in academia include extension decision-making theory, decision tree, behavioral decisionmaking theory, and multiobjective decision-making:

(1) Extension decision-making mainly has high requirements for the existing strategy generation, conversion bridge method, optimization evaluation method, and extension analytic hierarchy process, but it has low matching degree with the airport taxi scheduling optimization problem.

(2) Decision-making based on decision tree requires decision-makers to have a clear decision-making scheme at every step, while taxi drivers do not have a strict and clear scheme on the way to carry passengers, so the decision tree scheme is difficult to apply.

(3) Behavioral decision theory is more valued by researchers. It has developed from the maximum expected utility theory based on rational people and puts forward heuristic deviation. However, in the airport taxi scheduling problem, it is difficult for drivers and passengers to be rational.

(4) Multiobjective decision-making needs to establish multiple objective optimization models to solve high-dimensional linear programming or even nonlinear programming, so it is difficult to obtain the optimal solution.

Combined with the practice of the above optimal decision-making problems, this paper makes field investigation and data analysis, establishes a decision-making model based on driver income, and calibrates the parameters. The model is accurate through the verification of actual data.

1.1. Literature Review. The problem of airport taxi carrying passengers is related to the external image of a city, and it is also a problem that plagues many large cities, especially megacities. It is mainly manifested in the insufficient capacity of airport taxis, and the arrival of passenger flow at the airport is greatly affected by the distribution of flight times. How to coordinate transportation capacity and match the flow of passengers is the core issue. There are some relevant basic researches at home and abroad: First, some scholars have done preliminary research on driver decision-making.
$\mathrm{Yu}$ [1] proposed that the probability that a vacant taxi is matched with a passenger during the traversal of a link is calculated based on temporal Poisson arrivals of passengers and spatial Poisson distributions of competing vacant taxis. Passenger destination probabilities are calculated directly using observed fractions of passengers going to destinations from a given origin. Yang [2] presented the space-time trajectory cube as a framework for dividing and organizing the trajectory space in terms of three dimensions (origin, destination, and time). After that, space-time trajectory cube computation and origin-destination constrained experience extraction methods were proposed to extract the finegrained experience of taxi drivers based on a data set of real taxi trajectories. Zhu [3] started from the factors that influence the taxi driver's choice plan, constructed App M model and ADAM model based on the collected data, established the driver choice plan, and verified the rationality of the built model with actual operating data. The algorithm only simply simulates the driver selection scheme based on benefit consideration and lacks consideration in the calculation method of driver benefit, and the selection of the method needs to be optimized. Yu [4] analyzed the relevant factors that affect the decision-making of taxi drivers, established a driver's decision-making model based on queuing theory, and gave the driver's corresponding selection strategy. Using queuing theory to discuss the choice of drivers, there are few factors considered. The choice of whether the driver chooses to pick up passengers at the airport or back to the urban area is a multifactor subjective decision-making issue that requires comprehensive analysis and weighing. In terms of balancing long-distance and short-distance benefits, Li [5] established a prediction model for passenger flow in the airport taxi ride area, established a deterministic decision-making model for drivers whether to wait or not, and used a goal planning model to solve the priority of short-distance for passengers. It provides ideas for solving short-distance drivers prioritizing recarrying passengers. It is not clear to judge and identify short-distance drivers, and further research is needed. Zhou [6] selected six influencing factors: number of flights, number of vehicles in storage pool, temperature, weather, time period, and holidays, and established multiple linear regression models and elastic network regression models to analyze and judge the driver's decision-making choices. The literature has few analyses on the accuracy and applicability of the model, and subsequent confirmatory studies are needed. Li [7] used regression model to establish the evaluation model of the overall service level of taxi pick up area and the mathematical model between service level and queuing time and divided the service level; the research can be used for the planning of taxi pick-up area facilities. The design provides a quantitative measure. Shi [8] aimed at the problem of setting up the taxi ride area at the airport and divided the pick-up process of the taxi in the ride area into three stages: vehicle seating, passenger boarding, and vehicle leaving. A simulation model for airport taxi transportation was established. Then, using Monte Carlo simulation, by comparing the passing times of unit vehicles under different conditions, the best setting plan of the airport taxi riding 
area is obtained, which can provide scientific and reasonable suggestions for the planning of the airport taxi riding area. The operating efficiency of the airport taxi delivery system is effectively improved. Yuan [9] constructed a taxi revenue function in the form of neuron s-characteristic function, introduced market parameters such as taxi market scale, waiting time, and price, discussed the supply-demand balance mechanism of taxi market, and constructed the taxi market optimization model with the goal of maximizing social welfare. Lee [10] used a set of hypothetical scenarios for air taxi network operation and evaluated the accuracy of the flow model against the outputs of the discrete-event model and illustrated how the flow model can be applied towards the optimal pricing of passenger fares for a single airport taxi service provider. Chen [11] proposed a multiview learning algorithm to extract a highly coupled and robust ship descriptor from multiple distinct ship feature sets and proposed a framework for integrating a multiview learning algorithm and a sparse representation method to track ships efficiently and effectively. Luo [12] analyzed three main factors affecting driving safety by using fuzzy theory, and the new calculation method of the reaction time was obtained. It can improve car-following safety model, which is established based on different reaction time. Cesar [13] introduced different operating restrictions on partial fulfilment of the operational targets as applied to zones of special interest or relating to the operation of the snowclearing machines. The problem is solved by optimization based on linear programming. Abdelghani [14] did a survey using the various methods to obtain the solution, which covers both theoretical and real AGAP with the description of mathematical formulations and resolution methods such as exact algorithms, heuristic algorithms, and metaheuristic algorithms.

The literature above has done basic research on the optimization of taxi capacity, established a mathematical model with certain reference significance, and made a detailed study on the optimization of airport and taxi scheduling. However, the taxi driver's income is not studied in combination with the taxi driver's selection strategy and airport flight factors, especially the airport lane layout and the design mode of carrying passenger, which will affect the taxi carrying efficiency.

From the above point of view, this paper studies the collaborative optimization of multiple factors, on the basis of ensuring the travel efficiency of passengers arriving at the airport and striving to maximize and balance the income of drivers.

1.2. The Focus of This Paper. This article mainly focuses on three aspects: (1) When faced with the choice of entering the airport to pick up passengers and entering the urban area, how to optimize the driver's decision and how to attract taxi drivers to the airport to ensure transportation capacity. (2) Optimize the passenger order of taxis at the airport and optimize the layout of the corridors for airport taxis to carry passengers. (3) Carry out the optimization plan design for reentry and passenger carrying for drivers who perform short-distance transportation tasks. The solution of the above problems will greatly promote the management and service level of airport taxis in mega cities.

\section{Problem Description}

At domestic airports, taxi drivers who drop off passengers to the airport are facing two choices to continue their route.

The first option is to go to the passenger arrival area and wait in line to pick up passengers. In particular, the taxi must wait in line at the designated "car storage pool," according to "first come, first out" rules queuing to enter the venue to pick up passengers, and the waiting time depends on the number of taxis in the queue and the number of passengers, which requires a certain time cost; the second option is to go back to the city with empty car to pick up passengers $[15,16]$, and the taxi driver will pay no-load charges and may lose the revenue of potential passengers. The choices for drivers to choose are shown in Figure 1.

The first option is called the A plan, and the second option is called the B plan. Then the problem of optimal allocation of airport taxi passengers can be transformed into the following subproblems:

(1) Mining the influence mechanism of related factors in taxi driver's decision-making. Before entering the airport, the driver can observe the number of flights arriving at the airport during a certain period of time and the number of vehicles currently in the "taxi storage pool. Based on how to determine the factors that affect the taxi driver's decision which are based on the relationship between the number of passengers and the income of taxi drivers, we established a taxi driver's choice decision model, studied the algorithm, and finally gave out the driver's choice strategy $[7,17,18]$.

(2) The management of passengers arriving at the airport involves two states [15]: the first one is that the passengers should queue and wait for taxis, and the other one is that taxis should queue for carrying passengers. How the airport management department designs taxi approach and passenger boarding schemes determined the pros and cons of the airport's refined management. Under the premise of ensuring the safety of people and vehicles, it can improve the efficiency of transportation and facilitate airport management.

(3) The income of airport taxis is directly related to the distance traveled by passengers, which cannot be known in advance. According to taxi management regulations, drivers cannot choose passengers or refuse to carry them, but taxis can travel back to the airport multiple times to carry passengers. How the management department implements a "priority" compensation plan for short-distance passenger taxis [19], so as to balance the benefits of short- 

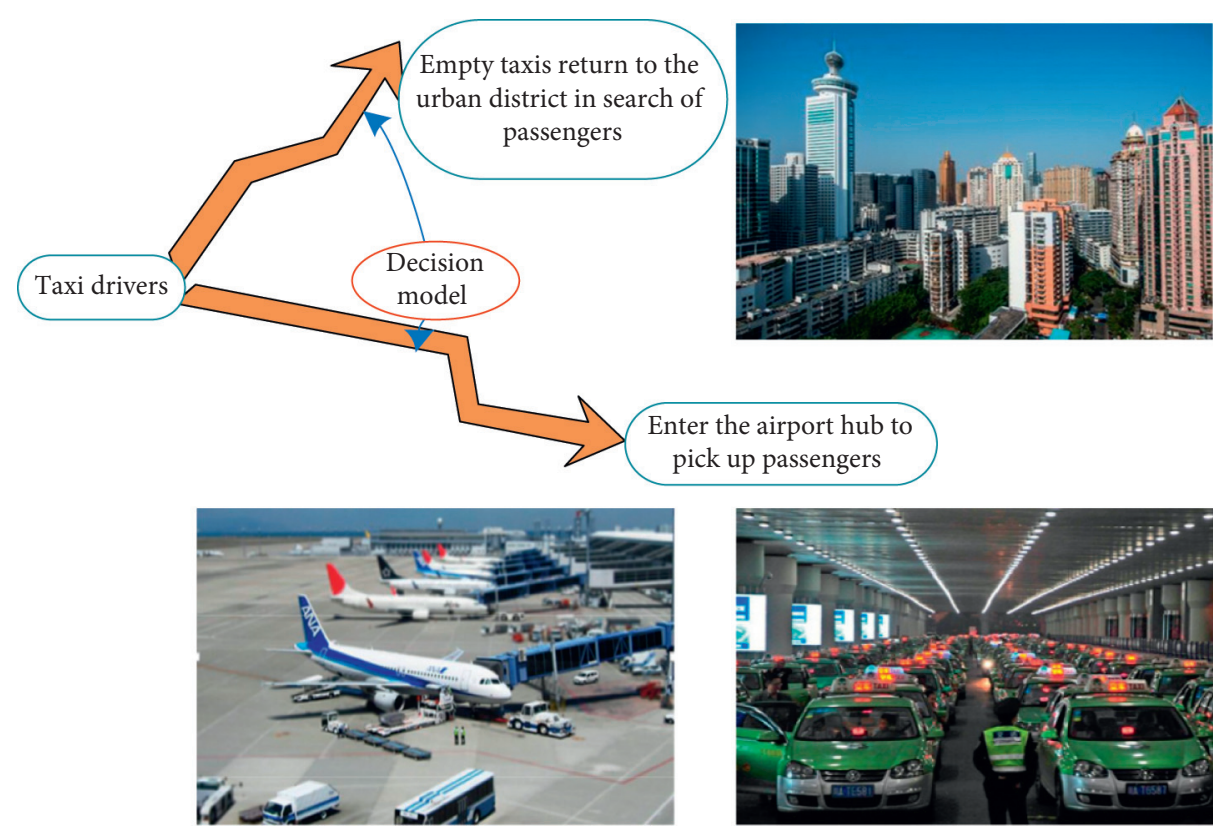

Figure 1: Taxi driver decision plan.

distance and long-distance taxis as much as possible, is of great importance.

The solution of the three above-mentioned core problems will finally solve the problem of the current shortage of airport taxis in mega cities and even the disorderly management and shorten the waiting time of passengers at the airport, in the meantime further enhancing the image of the city and society.

\section{Model Symbol Description and Conditional Assumptions}

3.1. Symbol Description. The model symbols used in the modeling process are drawn uniformly in Table 1, and the symbol modeling will be used directly as in the following.

3.2. Conditional Assumptions. To study the three problems decomposed above, we need to make the following assumptions based on actual business to quantitatively study the parameters and optimization problems in the model:

(1) It is assumed that the driver will immediately look for the next task after completing a task of transporting passengers.

(2) It is assumed that passengers can board the taxi immediately when the taxi stopped. It can guarantee not to waste time.

(3) It is assumed that the taxi driver will not return to the airport to continue to carry passengers after experiencing a long-distance passenger journey.

(4) It is assumed that the taxi driver cannot know the passenger's destination in advance.

\section{The Influencing Factors and Mechanism Mining of Taxi Driver's Decision}

4.1. Analysis of Factors Affecting Driver's Decision. After arriving at the airport, the main factors that affect drivers' choice are as follows [20]: the number of flights at that time and the time required to find passengers again. The time spent by the driver can be regarded as a uniform change at a constant speed. A high return per unit time of a driver means a high overall return. The driver's decision can be studied by comparing the average time return of each order of feasible solutions.

The number of flights mainly affects the waiting time of the drivers at the airport. If the number of passengers who arrive at the airport during a certain period and choose to take a taxi is less than the number of passengers in the queue, the time cost of drivers will increase, which may cause taxis to decrease. For the passenger searching time, it mainly affects the completion time of each order in scheme B. The driver searching time is different in different periods. Therefore, the GPS data set of taxis around the airport can be obtained through GPS positioning. This data set contains the driving route of taxis near the airport. The driving route is mostly determined by daily passenger experience. Drivers tend to drive to areas with more passengers or OD areas where passengers often get on. If there are more taxi travel demands in a certain range, it indicates that the range is an area with dense passenger demand [21-23]. The $K-$ Means clustering algorithm is used to process the collected GPS data, and then the hot area is obtained, and the distance between the hot passenger area and the airport is measured and averaged to obtain the average passenger-seeking distance for the driver in the urban area, and then the searching time can be obtained. 
TABLE 1: Modeling symbols and meanings.

$n$ : the number of flights the driver arrives at the airport

$N$ : the number of cars in the tank when the driver arrives at the airport $W_{i}$ : drivers choose to pick up passengers from the airport, the average revenue per order

$W_{j}$ : the average return of a driver who chooses to return to the urban area to carry passengers

$C_{i}$ : the average cost of a single order for the driver to pick up passengers from the airport

$C_{j}$ : the average cost of a single ticket when the driver chooses to return to the urban area to carry passengers

$D_{i}$ : driver's average passenger distance at the airport

$D_{j}$ : average passenger distance of the driver in the urban area

$T_{i}$ : the time it takes for the driver to complete a single order from the airport

$\delta$ : the average number of passengers carried on each flight

$\alpha$ : percentage of passengers choosing to take a taxi

$a_{1}$ : long-distance revenue

$a_{2}$ : short-distance revenue

$b_{1}$ : long-distance fuel consumption

$b_{2}$ : short-distance fuel consumption cost

$b_{0}$ : fuel consumption cost per kilometer

$t_{1}$ : waiting time in the storage tank

$t_{2}$ : one-time pick-up time for long-distance buses
$T_{j}$ : the time it takes for the driver to complete a single order in the city

$T$ : average searching time

$v$ : the speed of taxi

$p$ : the price of fuel consumption per kilometer of the taxi

$t$ : the average pick-up time at the airport

$C_{\mathrm{k}}$ : cost of one kilometer

$X$ : taxi GPS data set

$S$ : average passenger-seeking distance

$r$ : the average number of passengers carried by each taxi at the airport

$k$ : the proportion of passengers who choose to take a taxi for the airport

$d$ : average distance from the airport to major landmark locations

$t_{3}$ : one-time pick-up time for short-distance vehicles

$t_{4}$ : one green channel time for short-distance vehicles

$N$ : the number of times that short-distance vehicles pick up passengers at the airport

$X$ : the critical distance when the short-distance and longdistance benefits are equal

$K$ : univariate linear coefficient of short-distance distance and short-distance cost

$V:$ the taxi speed in the suburbs of the city

$\varepsilon$ : the passenger capacity of different models
The driver's choice of the two options is mainly determined by the economic benefits of the next order $\mathrm{A}$ or $B$. However, for the economic benefits brought by options $A$ and $\mathrm{B}$, the judgment criteria cannot be considered solely on the benefit of the next order. In fact, there are still two situations.

Option A: Although the carrying distance (hereinafter referred to as the carrying distance) is large and the single income is large too, it will be affected by the number of flights, and the waiting time will be longer, which will affect the income per unit time.

Option B: Although there is no waiting time, it needs time to search for passengers and the travel distance will have a greater impact on the revenue in this situation.

Therefore, a judgment and decision model can be constructed based on the number of flights and the passengers in the storage pool, and the clustering algorithm can be used for model and solve the average searching time from the airport based on the GPS data of the taxi. We make comparison and judgment on the income of the driver and finally establish a driver selection decision-making model.

4.2. Calculation of Average Passengers-Seeking Time Based on $K$ - Means Clustering. Collecting GPS data sets $X$ of taxis near the airport, the $K$-Means clustering algorithm is used for the cluster analysis of taxi searching for passengers, and the specific algorithm steps for establishing the clustering model are as follows.
Step 1. The $k$ initial clustering centers selected from the data set $X=\left\{x_{i} \mid x_{i} \in R P, i=1,2, \ldots, n\right\}$ were recorded as $m_{1} m_{2} \ldots \ldots, m_{i}$.

Step 2. Calculate the distance between each data point to be classified and the cluster center according to the mean value of each clustered data point, and redivide the corresponding data points according to the principle of minimum distance. The division is based on the Euclidean distance, as shown in the following formula:

$$
d\left(x_{i}, x_{j}\right)=\sqrt{\sum_{k=1}^{d}\left(x_{i k}-x_{j k}\right)^{2}} .
$$

Step 3. Recalculate the mean value of each cluster after classification. The calculation method is given below, where $n$ represents the $k-t h$ class inner vector:

$$
m_{t}=\frac{1}{n_{i}} \sum_{j=1}^{n_{i}} m_{i j}, i=1,2, \ldots \ldots, k .
$$

Step 4. Calculate the evaluation criterion function value $T$, which represents the sum of the individual error squares of all data center points, $P$ is the data point in the space, and the cluster center point is represented by $M$. The results generated according to the judgment criterion gradually tend to be independent and convergent. 


$$
T=\sum_{i=1}^{k} \sum_{p \in x_{i}}\left(p-m_{j}\right)^{2} .
$$

Step 5. Repeat Step 2 to Step 4 until the evaluation criterion function value $T$ stabilizes, so that the error sum of squares criterion function reaches the minimum; if the error sum of squares criterion function reaches the minimum, the algorithm converges and the calculation is completed. Otherwise, return to Step 2 and proceed to the next iteration.

The algorithm flow is shown in Figure 2.

Finally, the hotspot of passenger area $x$ can be obtained, and get the average passenger searching distance $s$ according to the weighted average of the distance between the passenger area and the airport; the average time $T$ of finding passengers is obtained finally.

\subsection{Construction of the Multiple Decision Model Based on} Multiple Parameters. The time required for the driver to choose plan A mainly includes the following: waiting time in the taxi storage tank $t_{i, 1}$, the time for each taxi to pick up passengers at the airport $t_{i, 2}$, and the time to deliver the passengers to the destination $t_{i, 3}$; the number of taxis in the storage tank in a certain period of time is $N, P_{i}$ is the average passenger-carrying mileage of a taxi, $D_{i}$ is the driver's choice to determine the average passenger-carrying distance under the plan, the cost of the driver's choice of plan A is set to $C_{i}, W_{i}$ is the taxi time price and congestion index, and $W_{A}$ represents the average income of plan A.

When plan A is selected, the total time spent by the driver is $T_{A}$, and the intensity of flight arrivals determines the number of passengers entering the taxi queuing system: when the number of flights is intensive, the number of passengers waiting to board the system exceeds the capacity of the taxis in the tank, and $T_{A}$ can be expressed as follows:

$$
T_{A}=N \times t_{i, 2}+t_{i, 3} .
$$

When there is no flight entering the port, the number of passengers waiting for taxi in the system is much less than the capacity of the taxi in the storage tank, and the taxi needs to wait in the storage tank; then $T_{A}^{\prime}$ is subjected to

$$
T_{A}^{\prime}=t_{i, 1}+N \times t_{i, 2}+t_{i, 3}
$$

Then, the average revenue per minute of the driver under plan $\mathrm{A}$ is given as follows:

$$
\begin{aligned}
W_{A} & =\frac{W_{i} \times D_{i} \times P_{i}-C_{i} \times D_{i}}{T_{A}} \\
\operatorname{or} W_{A}^{\prime} & =\frac{W_{i} \times D_{i} \times P_{i}-C_{i} \times D_{i}}{T_{A}^{\prime}} .
\end{aligned}
$$

When a driver chooses plan $\mathrm{B}$, the total cost time is $T_{B}$; if the intensive degree of flight arrivals is low, the drivers tend

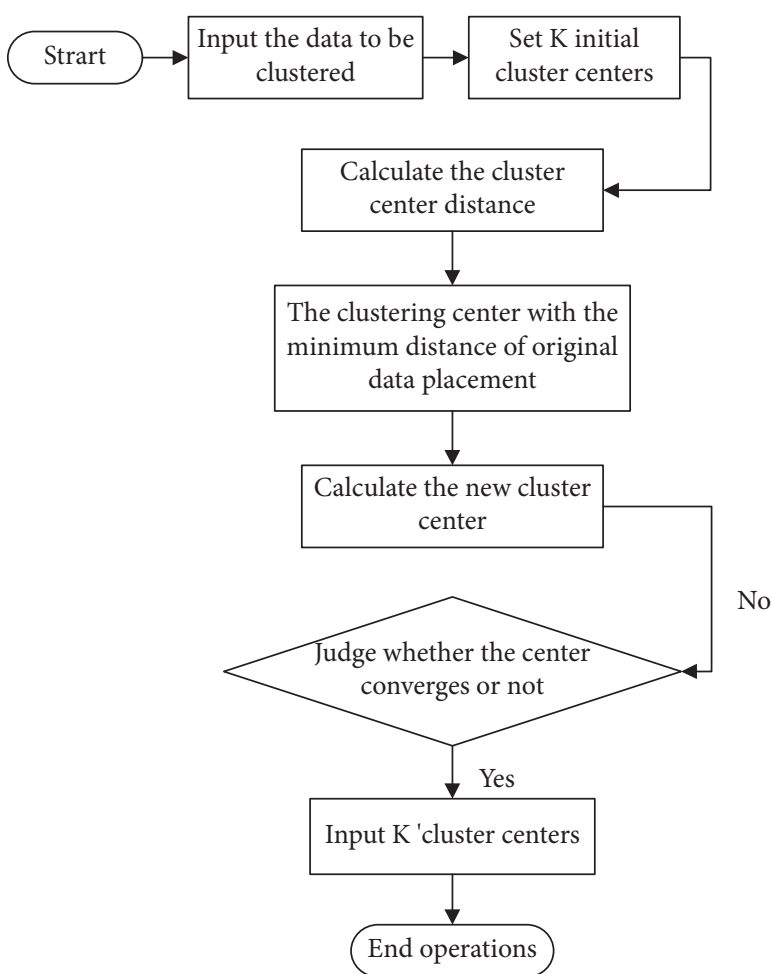

FIGURE 2: The process of determining the cluster center point.

to choose plan B. In this situation, the taxi capacity is much greater than the passenger demand, and the drivers choose to leave the airport to carry passengers into the urban area with densely packed passengers; set the passenger-seeking time as $t_{j, 1}$ and the passenger-carrying time as $t_{j, 2} . T_{B}$ can be expressed as follows:

$$
T_{B}=t_{j, 1}+t_{j, 2}
$$

The speed at which taxis look for passengers in an unloaded state is usually slow, denoted as $v_{e}$. The speed of passenger-carrying operation is faster, denoted as $v_{f}$; that is, $v_{f} \geq v_{e} . t_{j, 1}$ and $t_{j, 2}$ are given as follows:

$$
\begin{aligned}
t_{j, 1} & =\frac{D_{j}}{v_{e}}, \\
t_{j, 2} & =\frac{D_{i}}{v_{f}} .
\end{aligned}
$$

When plan B is selected, substituting formula (8) into the drivers' average income $W_{B}$ per unit time under plan is shown as follows:

$$
\begin{aligned}
W_{B}= & \frac{D_{i} \times P_{i}-D_{j} \times C_{k}-D_{i} \times C_{i}}{T_{B}} \\
= & \frac{D_{i} \times\left(P_{i}-C_{i}\right)-D_{j} \times C_{k}}{D_{j} / v_{e}+D_{i} / v_{f}} .
\end{aligned}
$$

Further simplify the derivation to obtain the following formula: 


$$
\begin{aligned}
W_{B}= & \frac{D_{i} \times\left(P_{i}-C_{i}\right)-D_{j} \times C_{k}}{D_{j} / v_{e}+D_{i} / v_{f}} \\
& =\frac{D_{i} \times\left(P_{i}-C_{i}\right)-D_{j} \times C_{k}}{D_{j} \times v_{f}+D_{i} \times v_{e}} \times v_{f} \times v_{e} .
\end{aligned}
$$

Thus, the driver's decision-making judgment criterion can be obtained by comparing $W_{A}$ and $W_{B}$ in equation (6) and equation (10) given as follows:

$$
\left\{\begin{array}{l}
\text { choose scheme } A, W_{A} \geq W_{B} \\
\text { choose scheme } B, W_{A} \leq W_{B}
\end{array}\right.
$$

The drivers can know the number of flight arrivals based on the granularity of the time period in advance, so the driver's decision can be determined based on the relationship between the average number of waiting passengers (hereinafter referred to as the demand) and the average taxi capacity during the time period (hereinafter referred to as the supply). The average number of waiting passengers in a given period can be obtained from the statistics of the number of flights and aircraft types given by the airport, and the average capacity can be obtained based on the number of taxis in the storage pool during the period.

\section{Decision-Making Strategy Modeling of Airport Taxi Drivers}

5.1. Collection of the Driver-Decision Model. According to the decision-making model constructed in Section 4.3, the taxi driver's decision-making strategy model is obtained by calibrating the parameters with the domestic airport data.

The taxi charging standards and average fuel consumption and driving distance of domestic airport taxis are shown in Tables 2 and 3, respectively.

According to the flight information of a certain domestic airport, the number of flights arriving at this airport is mainly affected by the time of day and whether it is a holiday. According to the collected flight data for every two hours of a domestic airport on working days, nonworking days, and holidays, the number of flight arrivals is recorded as $n$; the number of flight arrivals at a certain domestic airport in different dates and time periods is shown in Table 4. According to survey data, the proportion of passengers who choose taxis after arriving at the airport is relatively stable. About $15 \%$ of passengers will choose taxis during the daytime and $45 \%$ at night. Table 4 and Figure 3 show the ratio of taxis at a certain domestic airport.

According to flight data, the demand for taxis in the time period is accurately estimated. The proportion of passengers who choose to take taxis in a certain time period is shown in Table 5 , and it can be expressed by the following equation:

$$
M=n \times \varepsilon \times \alpha,
$$

where $n$ is the number of taxi drivers arriving at the airport; $\varepsilon$ is the passenger capacity of different models; and $\alpha$ is proportion of taking a taxi.
TABLE 2: Taxi charging standard in a city.

\begin{tabular}{lcc}
\hline Kilometers & Day $(5: 00-23: 00)$ & Night $(23: 00-5: 00)$ \\
\hline $0 \sim 3 \mathrm{~km}$ & $14 \mathrm{RMB}$ & $18 \mathrm{RMB}$ \\
$3-15 \mathrm{~km}$ & $2.5 \mathrm{RMB} / \mathrm{km}$ & $3.1 \mathrm{RMB} / \mathrm{km}$ \\
More than $15 \mathrm{~km}$ & $3.6 \mathrm{RMB} / \mathrm{km}$ & $4.7 \mathrm{RMB} / \mathrm{km}$ \\
\hline
\end{tabular}

TABLE 3: Average fuel consumption and driving distance of taxis in an airport of a city.

\begin{tabular}{lc}
\hline Average fuel consumption per kilometer $(\mathrm{RMB} / \mathrm{km})$ & 0.44 \\
\hline Average driving distance $(\mathrm{km})$ & 60 \\
\hline
\end{tabular}

TABLE 4: The flights arrival in different periods of a certain domestic airport.

\begin{tabular}{lccc}
\hline Observation period & Weekday & Weekend & Holiday \\
\hline 23:00-01:00 & 4 & 6 & 9 \\
01:00-03:00 & 2 & 5 & 8 \\
03:00-5:00 & 4 & 8 & 12 \\
05:00-7:00 & 12 & 14 & 19 \\
07:00-9:00 & 19 & 18 & 31 \\
09:00-11:00 & 24 & 28 & 38 \\
11:00-13:00 & 20 & 31 & 40 \\
13:00-15:00 & 23 & 23 & 34 \\
15:00-17:00 & 15 & 13 & 23 \\
17:00-19:00 & 16 & 12 & 38 \\
19:00-21:00 & 18 & 18 & 26 \\
21:00-23:00 & 9 & 15 & 19 \\
\hline
\end{tabular}

5.2. Calculation of Airport Passenger Revenue. The income of the driver who chooses to wait for passengers at the airport is mainly related to the average passenger-carrying distance and the waiting time in the storage tank. However, it is difficult to obtain the average passenger distance data of airport taxis [24]. According to the operating data of the four major taxi companies in a certain city, the passenger flow distribution law of the city's airport passengers, the distribution weight of the passenger flow, and the travel characteristics of different passenger flows are estimated to obtain the average passenger-carrying distance for different characteristic dates.

The passenger flow of the airport is mainly divided into two parts: tourist flow and other passengers flow. The passenger flow is different in different periods, and the destinations that different passenger flows choose to go to by taxi also have a large gap, so the benefit is different too. The passenger flow ratio of the airport in different periods is shown in Table 6.

Tourists choose taxis mainly to go to scenic spots or hotels, while residents are familiar with the local traffic conditions and will choose to take taxis at a relatively short distance [25]; airport taxi mileage data are collected from four major taxi operating companies, and the weighted average of taxi operating mileage is taken and used as the average passenger-carrying distance of the tourist flow, denoted as $x_{1}$; the average travel distance of the resident passenger flow is recorded as $x_{2}$, the average passengercarrying distances of taxis for different passenger flows on different characteristic days of a certain airport are obtained, as shown in Table 7. 


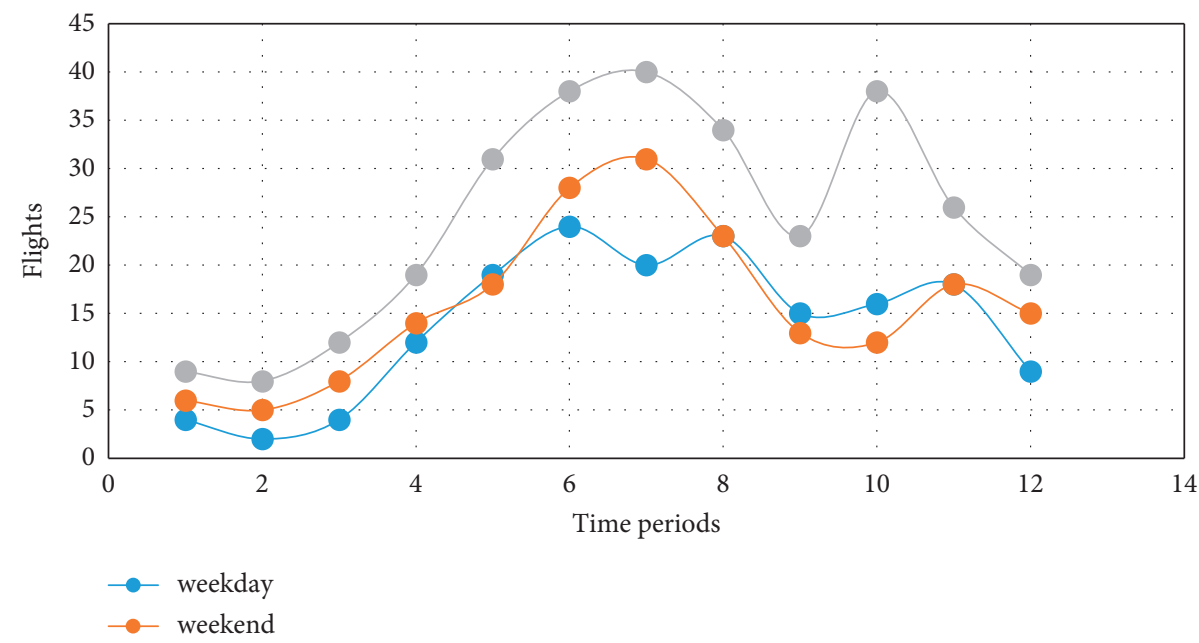

FIGURE 3: Flights arrivals in different periods.

Table 5: Proportion of passengers choosing taxis in Pudong Airport.

\begin{tabular}{lcc}
\hline Category & Daytime (\%) & Night (\%) \\
\hline Proportion of taking taxi $(\alpha)$ & 15 & 45 \\
\hline
\end{tabular}

TABle 6: Percentage of passenger flow at an airport with different daily characteristics.

\begin{tabular}{lccc}
\hline Category & Weekday & Weekend & Holiday \\
\hline Tourist flow $\left(\partial_{1}\right)$ & 0.44 & 0.51 & 0.65 \\
Other passengers flow $\left(\partial_{2}\right)$ & 0.56 & 0.49 & 0.35 \\
\hline
\end{tabular}

TABLE 7: Average carrying distances of taxis with different passenger flows on different days $(\mathrm{km})$.

\begin{tabular}{lccc}
\hline Carrying distances & Weekday & Weekend & Holiday \\
\hline Tourist flow $\left(\partial_{1}\right)$ & 24.52 & 25.68 & 27.62 \\
Other passengers flow $\left(\partial_{2}\right)$ & 16.45 & 18.24 & 20.85 \\
\hline
\end{tabular}

According to Table 8, the average passenger-carrying distance of different passenger flows in different periods is measured and averaged, and the average carrying distance of each passenger at the airport of the driver is calculated as follows:

$$
D_{i}=x_{1} \times \partial_{1}+x_{2} \times \partial_{2}
$$

Therefore, the average driver's income per order $W_{i}$ can be obtained as follows:

$$
W_{i}=D_{i} \times p_{i}
$$

Before entering the storage tank, the driver can know the number of taxis in the storage tank in advance. Through the investigation and observation, the average time for passengers to board the taxi is $t=0.5 \mathrm{~min}$, so the average waiting time $t_{1}$ of the driver in the storage tank can be calculated as follows:
TABle 8: Average carrying distances of taxis on different time periods $(\mathrm{km})$

\begin{tabular}{ll}
\hline Time periods & Distance \\
\hline Weekday & 21.95828 \\
Weekend & 30.07744 \\
Holiday & 40.47122 \\
\hline
\end{tabular}

$$
t_{1}=\left\{\begin{array}{l}
0.5 \times N \\
0.5 \times N+\Delta t
\end{array} .\right.
$$

$\Delta t$ is the waiting time in the taxi storage tank.

According to the average fuel consumption per kilometer $C_{i}$ of a certain city and the average passenger-carrying distance $D_{i}$ of the driver at the airport, the average cost $C$ of the driver choosing to carry passengers from the airport is obtained as follows:

$$
C=C_{i} \times D_{i}
$$

Calculate the average passenger-carrying time $t_{2}$ based on the average speed of local taxis as follows:

$$
t_{2}=\frac{D_{i}}{v}
$$

Add the two times to get the average time $T_{i}$ per order which is shown as follows:

$$
T_{i}=t_{1}+t_{2}
$$

Finally, the income of picking up passengers at the airport can be shown as follows:

$$
\begin{aligned}
W_{A} & =\frac{W_{i}-C}{T_{i}} \\
& =\frac{D_{i} \times p_{i}-C_{i} \times D_{i}}{D i / v+0.5 N} .
\end{aligned}
$$


5.3. Calculation Income of Carrying Passengers in the Urban Area. There is a big difference in the calculation method of income between the carrying passenger in urban area and waiting for passengers in the storage tank [26]. In the former mode, there is no waiting time for passengers in the airport storage tank, but the empty search distance and time are extra. In the calculation process, assuming that the driver searches for the next order immediately after completing one, the passenger-seeking distance is the empty driving distance (empty driving distance).

In this mode, the average passenger-carrying distance and empty driving distance need to be obtained to calculate the average revenue per order. According to the taxi operation data of a certain city, the average passenger-carrying distance in different periods can be obtained as $D_{j}$, as is shown in Table 9.

According to the $K$-Means clustering algorithm, the average passenger-seeking time is calculated. The passengerseeking distance is the empty driving; however, the empty driving distance in different periods is different, so the empty driving distance can be converted according to the mileage utilization rate in each period, which is shown in Table 10.

Thus, the relationship between average empty driving distance and average passenger-seeking distance $k_{\text {length }}$ can be given by formula (20), and the average passenger-seeking distance in different time periods is shown in Table 11.

$$
k_{\text {length }}=u \times y_{\text {length- }} y_{\text {length }} \text {. }
$$

Here, $\mu$ is mileage utilization; $y_{\text {length }}$ is operating mileage; total $_{\text {length }}$ is the total mileage driven; $k_{\text {length }}$ is empty driving distance; total $l_{\text {length }}=y_{\text {length }}+k_{\text {length }} ; \mu=y_{\text {length }} /$ total $_{\text {length }}$.

According to the average speed $V$ of a taxi, time $T_{j}$, cost $C_{j}$, and income $w_{j}$ for the driver to complete a single order in the urban area are calculated.

$$
\begin{gathered}
T_{j}=\frac{\left(D_{j}+s\right)}{v} . \\
C_{j}=\left(D_{j}+s\right) \times c .
\end{gathered}
$$

According to formula (21) and formula (22), the average cost of a single order $w_{j}$ for drivers who choose to return to the urban area to pick up passengers is shown as follows:

$$
\begin{aligned}
w_{j} & =\frac{D_{j}-c_{j}}{T_{j}} \\
& =\frac{D_{j} \times p-\left(D_{j}+s\right) \times c}{D_{j}+s / v} .
\end{aligned}
$$

\section{Optimization of Taxi Passenger-Carrying Order and Layout}

6.1. Analysis of Taxi Queue of Passenger Carrying Based on Queuing Theory. In order that taxis can serve passengers arriving at the airport efficiently, the queuing theory is proposed to optimize taxi queuing and passengers boarding process [27]. Assuming that there is no upper limit on the
TABle 9: Average carrying passenger distance of taxis in Shanghai in different periods $(\mathrm{km})$.

\begin{tabular}{lc}
\hline Time distribution & Average passenger-carrying distance \\
\hline Weekday & 8.67 \\
Weekend & 8.28 \\
Holiday & 9.14 \\
\hline
\end{tabular}

TABLE 10: Mileage utilization at different time periods of the operating day.

\begin{tabular}{lc}
\hline Time period & Mileage utilization (\%) \\
\hline 1:00 3:00 & 48 \\
3:00 5:00 & 38 \\
5:00 7:00 & 42 \\
7:00 9:00 9:00 11:00 & 54 \\
11:00 13:00 & 66 \\
13:00 15:00 15:00 17:00 & 60 \\
17:00 19:00 & 64 \\
19:00 21:00 $1: 00 \sim 23: 00$ & 58 \\
23:00 1:00 & 64 \\
\hline
\end{tabular}

TABle 11: Average passenger-seeking distance in different time periods.

\begin{tabular}{lccc}
\hline Time period & Weekday & Weekend & Holiday \\
\hline 1:00 3:00 & 9.39 & 8.97 & 9.90 \\
3:00 5:00 & 14.14 & 13.51 & 14.91 \\
5:00 7:00 & 11.97 & 11.43 & 12.62 \\
7:00 9:00 & 7.38 & 7.05 & 7.79 \\
9:00 11:00 & 4.47 & 4.27 & 4.71 \\
11:00 13:00 & 5.78 & 5.52 & 6.09 \\
13:00 15:00 & 4.88 & 4.66 & 5.14 \\
15:00 17:00 & 6.28 & 6.00 & 6.62 \\
17:00 19:00 & 4.88 & 4.66 & 5.14 \\
19:00 21:00 & 8.00 & 7.64 & 8.44 \\
21:00 23:00 & 8.67 & 8.28 & 9.14 \\
23:00 1:00 & 9.78 & 9.43 & 11.23 \\
\hline
\end{tabular}

number of passenger boarding points, a taxi is arranged at each boarding point by default, and the boarding points are evenly distributed in the airport boarding area, and the distance between passengers arriving at each boarding point is the same; the optimization problem conforms to the $M / M / S$ queuing system.

The goal of optimization is to ensure that passengers are evacuated quickly, and the optimal number of waiting points is calculated through modeling so as to minimize the cost of the reception and the loss of passengers due to waiting. The number of passengers arriving at the waiting point can be obtained from the flight information; calculate the reception cost and the loss of passengers waiting. From the relationship between the queue length $L$, the service intensity $\rho$, and the number of receptions $S$ in the M/M/S model, the functional dependence of $L$ on $S$ is estimated, and the optimal solution of the built model $S$ is calculated finally, that is, the location and opening number of the optimal boarding point. 
It is proposed to design a "priority" scheme for drivers with short carrying distance to enter the airport to carry passengers, so as to avoid serious imbalance in benefits due to the large difference in passenger-carrying distance. To solve the problem, the following optimization model is proposed. According to equations (9)-(11), the optimal model of driver benefit balance based on the passengercarrying distance can be transformed into the minimum absolute value of the short-distance taxis and long-distance taxis in the same time.

The calculation model of the average revenue of long and short passenger-carrying distances, respectively, is given, and the actual operating data is used to verify and compare the average return value, and they are discussed under the extreme conditions, so as to obtain a critical mileage value that distinguishes priority release. In addition, we compare the critical point with the numerical deviation rate and evaluate the rationality of the model. To make the evacuation rate of passengers higher and the queuing time as short as possible, we require as many boarding points as possible. At the same time, we need to consider that each boarding point is used reasonably and its construction and maintenance costs need to be as low as possible; therefore, the utilization rate $\rho$ of the boarding point should reach a reasonable value.

6.2. Optimization Plan of Airport Layout Design. Taking into account the safety factors of boarding passengers at the airport, if two lanes are queued for boarding passengers, passengers must cross the inner lane when boarding from the outer lane, which poses a greater safety hazard. At the same time, passengers tend to prefer to leave the inner lane, leading to the outer lane low utilization efficiency. Based on this, the paper assumes that it is a one-sided lane to board passengers; the side of the lane near the airport entrance and exit is the location of the passenger boarding point. The outer lane is the passage for the taxi to enter and leave the boarding point. In addition, since the boarding points are evenly distributed in the boarding area, passengers arrive at each boarding point with the same distance, so there is no need to consider the influence of the location of the pick-up point. Therefore, the influence of the location of the boarding point is not considered. The exits and boarding points of airport are shown in Figure 4.

6.3. Linear Programming Model Based on Queuing Theory. Based on queuing theory, the $M / M / S$ model for setting up the entire boarding point is a Poisson input, negative exponential distribution service, and multiple service desks. When there are people queuing at the exit, the taxi queuing system starts to enter the busy period. Passengers in the system are in a queue waiting state or go to the corresponding pick-up point from the exit to get on the car; once the queue and the number of people on the car is 0 , the system enters the idle period. The utilization rate of the service desk (pick-up point) of the queuing system is denoted by $\rho$, which represents the service strength of the queuing system. In the $M / M / S$ model,
$S$ is the number of points on board; $\mu$ is the number of passengers served per unit time; $\lambda$ is the average number of passengers arriving at the system per unit time, that is, the arrival rate.

For the queuing system, the main indicators for evaluating its pros and cons are queuing length and customer stay time. From the perspective of optimization, the two need to be shortened as much as possible. The actual queuing process can be simplified, and the passenger queue can be defined as a single line. When a taxi arrives at the boarding point, the corresponding number of passengers will be released to the simplified model.

In order to determine the amount of the optimal boarding points in the case of $\lambda$ and $\mu$, it is necessary to calculate the probability $p_{i}$ of $i$ passengers in the system under the condition of statistical equilibrium.

$$
\begin{gathered}
p_{0}=\left(\sum_{i=1}^{s-1} \frac{(s \rho)^{i}}{i !}+\frac{(s \rho)^{s}}{s !} * \frac{1}{1-\rho}\right)^{-1} . \\
p_{i}= \begin{cases}\frac{(s \rho)^{i}}{i !} p_{0}, & 1 \leq i \leq s \\
\frac{s^{s} \rho^{i}}{s !} p_{0}, & i \leq s\end{cases}
\end{gathered}
$$

Taking into account the actual situation of airport construction, $S$ cannot be too large. When all the pick-up points are occupied, the probability $D$ that passengers need to wait can be expressed as follows:

$$
D=1-\sum_{i=0}^{s-1} p_{i} .
$$

Seek captain $L_{s}$ 's expectation $L_{q}$ as shown in the three following formulas:

$$
\begin{array}{r}
L_{s}=\sum_{i=0}^{\infty} i \times p_{i}=s \rho+\frac{\rho}{(1-\rho)^{2}} p_{s} \\
L_{q}=\sum_{i=0}^{\infty} i \times p_{s+i}=\frac{\rho}{(1-\rho)^{2}} p_{s} \\
\text { so: } L_{s}=L_{q}+s \rho \\
=\frac{1}{s !} * \frac{(\rho s)^{s}}{(1-\rho)^{2}} p_{0}+\frac{\lambda}{\mu}
\end{array}
$$

Because passengers are unable to leave the airport or conduct other personal businesses while waiting in the queue, passengers should be deemed to have corresponding losses during the waiting time, and the loss per unit time (hour) of passengers is converted into economic losses $\alpha$. The construction and maintenance costs are $\beta$, and the total cost is $s * \beta$.

Therefore, evacuating passengers as soon as possible and ensuring the highest riding efficiency can be transformed into finding the minimum sum of the losses caused by waiting for passengers and the construction and 


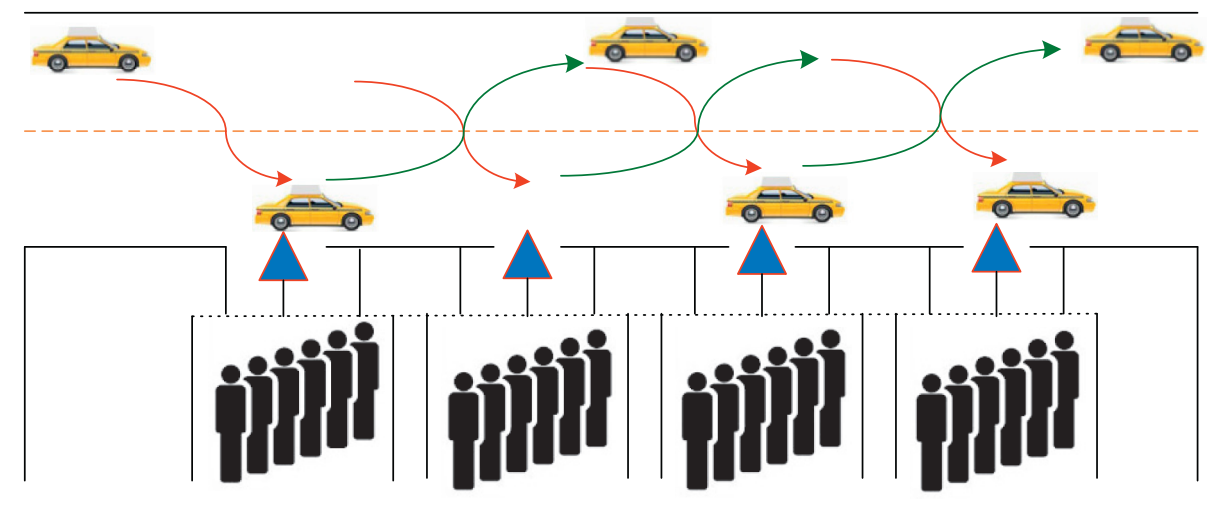

FIgure 4: Taxi points in the boarding area.

maintenance cost of the boarding point, which can be transformed into the following objective function:

$$
\min Z(s)=z_{1}+z_{2} .
$$

Here, $z_{1}=L s \times \alpha$ is the loss of passengers for waiting; $z_{2}=s * \beta$ is the construction cost and maintenance cost of setting up a pick-up point for the airport. The objective function is shown as follows:

$$
\left\{\begin{array}{l}
Z(s) \leq Z(s+1) \\
Z(s) \leq Z(s-1)
\end{array} .\right.
$$

Constraints can be transformed as shown in the following formula:

$$
Z(s-1)-Z(s) \geq \frac{\alpha}{\beta} \geq Z(s+1)-Z(s) .
$$

Finally, finding the optimal solution $S_{m}$, because $S_{m}$ is the optimal solution, there are $Z\left(S_{m}+1\right) \geq Z\left(S_{m}\right)$ and $Z\left(S_{m}-1\right) \geq Z\left(S_{m}\right)$. The arrival rate of the passenger flow $\lambda$ collected at an airport and the number of people $\mu$ boarding the train per unit time are substituted into the constructed optimization model to solve the optimal solution.

\section{Numerical Experiments}

\subsection{Model Solving and Rationality Analysis}

7.1.1. The Acquisition Scheme of Total Passenger Flow at the Airport

(1) Location of investigation: Shang Hongqiao Airport

(2) Investigators: 20 postgraduates

(3) Survey time: May 28, 2020, to June 3, 2020

According to the characteristics of airport operation period, 12 time periods are divided, which can be calculated by investigating the arrival of the airport flight, so the passenger quantity with travel demand after the flight arrives at the airport can be calculated.

7.1.2. The Threshold of Driver Decision. According to the airport data collected in Step 1, the model is established by using MATLAB, and the results are shown in Table 12.
According to the data in Table 12, in a certain period (1 : 00-3:00) (working day), when the number of taxis in the storage pool reaches a certain value (58), the driver will choose to go to the urban area to pick up passengers, and the income will be higher, which can be shown in Figure 5 .

7.2. The Solution of the Model. By substituting the flight data of an airport collected earlier into the model, the number of passengers arriving at the loading area every two hours at each exit can be obtained from Table 13.

At the airport, the average number of passengers per taxi is $r=1.5$, and the passenger flow of each boarding point every two hours is calculated to be 360 . Two hours is defined as the unit time, and $\lambda$ is the average number of passengers arriving in the unit time; that is, the average arrival rate is $\lambda$ $=360$ passengers $/ 2 \mathrm{~h}$, and $\mu$ is the average number of passengers who have completed the service in a unit time. Through investigation, the ratio of passenger time cost $\alpha$ to construction cost and maintenance cost $\beta$ of a single boarding point is $1: 400$.

Take the above data into equation (29) to equation (32): calculate the values of $s$, and arrange them according to time period and characteristic day; we can get the number of boarding points opened in different periods of time in an airport which is shown in Table 14 .

Determine the number of open pick-up points under different passenger flow, and then put the vehicles and passengers into the receiving area and loading area in a certain number of batches.

The boarding points needed are shown in Figure 6.

\section{Optimization of Reloading Order and Sensitivity Analysis}

8.1. Optimization of Reloading Order Based on Short Distance. From the perspective of balancing the interests of taxi drivers, it is necessary to give priority to drivers carrying short-distance passengers to return to the airport to pick up passengers again.

It is proposed to establish a green channel for shortdistance drivers, so that all qualified short-distance drivers do not need to enter the vehicle storage pool for waiting but 
TABLE 12: Driver selection decision scheme in different periods.

\begin{tabular}{lccc}
\hline Observing session & Working days (persons) & Nonworking days (persons) & Holiday (persons) \\
\hline 1:00 3:00 & 58.8 & 119.6 & 152.2 \\
3:00 5:00 & 130.8 & 163.4 & 206.4 \\
5:00 7:00 & 62.2 & 70.8 & 84.5 \\
7:00 9:00 & 3.3 & 45.0 & 54.3 \\
9:00 11:00 & 11.3 & 28.7 & 35.1 \\
$11: 00 \sim 13: 00$ & 31.3 & 36.0 & 43.7 \\
13:00 15:00 & 26.8 & 30.9 & 37.8 \\
15:00 17:00 & 33.8 & 38.8 & 46.9 \\
17:00 19:00 & 26.8 & 30.9 & 37.8 \\
19:00 21:00 & 6.4 & 48.4 & 58.3 \\
$21: 00 \sim 23: 00$ & 9.7 & 16.2 & 62.7 \\
$23: 00 \sim 1: 00$ & 61.7 & 87.2 & 120.5 \\
\hline
\end{tabular}

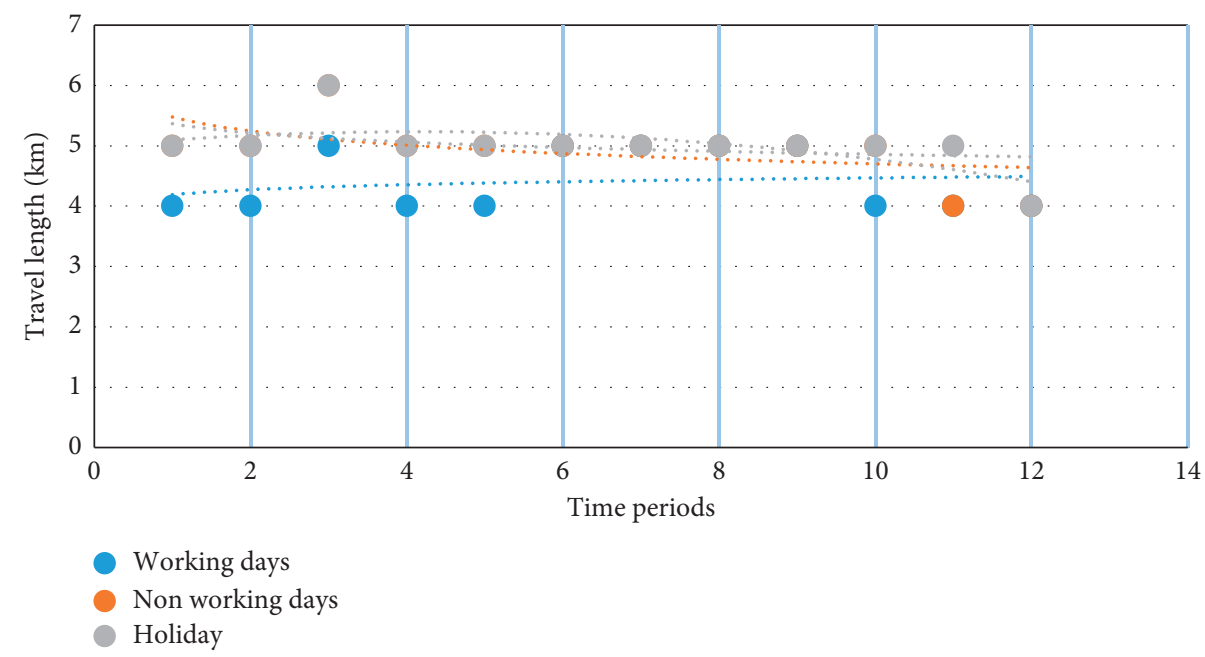

FIGURE 5: Driver selection decision scheme in different periods.

TABle 13: Passengers arriving at the loading area within unit time of an airport in different periods.

\begin{tabular}{lccc}
\hline Observing session & Working days & Nonworking days & Holiday \\
\hline $23: 00-1: 00$ & 420 & 540 & 540 \\
$1: 00-3: 00$ & 630 & 630 & 630 \\
$3: 00-5: 00$ & 540 & 720 & 720 \\
$5: 00-7: 00$ & 420 & 480 & 480 \\
$7: 00-9: 00$ & 420 & 540 & 540 \\
$9: 00-11: 00$ & 480 & 600 & 600 \\
$11: 00-13: 00$ & 540 & 600 & 660 \\
$13: 00-15: 00$ & 540 & 540 & 660 \\
$15: 00-17: 00$ & 480 & 480 & 600 \\
$17: 00-19: 00$ & 420 & 420 & 540 \\
$19: 00-21: 00$ & 400 & 380 & 480 \\
$21: 00-23: 00$ & 400 & & 420 \\
\hline
\end{tabular}

queue up in the green channel to receive passengers; the operation mode of green channel is given below.

Step 6. Before starting to use the green channel, taxis waiting to receive passengers at the airport should wait $t_{1}$ in the parking pool.

Step 7. When a taxi receives a short-distance order, it can return to the green channel of the airport when completing the order and will be given the priority to waiting for passengers; when a taxi receives a long-distance order, even when it returns to the airport, it needs to enter the parking pool to queue up, instead of taking the green channel. Due to the large distance, high time cost, and high fuel consumption, drivers who receive long-distance orders may not choose to return to the airport to rearrange the team for $t_{1}$ to pick up the next passenger but choose to solicit passengers nearby. 
TABLE 14: The number of boarding points opened in different periods in the airport.

\begin{tabular}{lccc}
\hline Observing session & Working days & Nonworking days & Holiday \\
\hline $23: 00-1: 00$ & 4 & 5 & 5 \\
$1: 00-3: 00$ & 4 & 5 & 5 \\
$3: 00-5: 00$ & 5 & 6 & 6 \\
$5: 00-7: 00$ & 4 & 5 & 5 \\
$7: 00-9: 00$ & 4 & 5 & 5 \\
$9: 00-11: 00$ & 5 & 5 & 5 \\
$11: 00-13: 00$ & 5 & 5 & 5 \\
$13: 00-15: 00$ & 5 & 5 & 5 \\
$15: 00-17: 00$ & 5 & 5 & 5 \\
$17: 00-19: 00$ & 4 & 5 & 5 \\
$19: 00-21: 00$ & 4 & 4 & 5 \\
$21: 00-23: 00$ & 4 & 4 & 4 \\
\hline
\end{tabular}

The number of boarding points opened

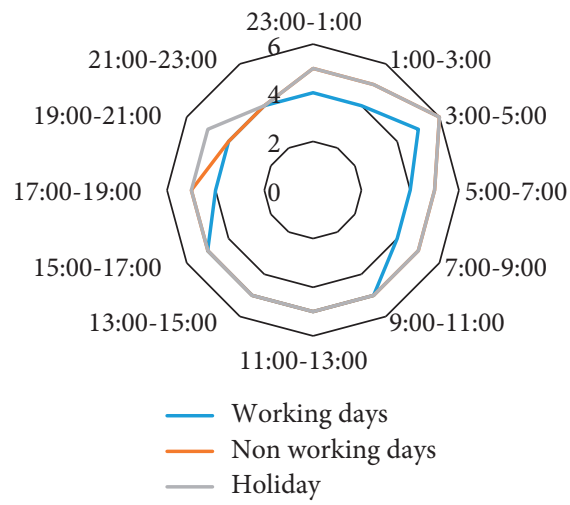

FIGURE 6: The number of boarding points opened in different periods.

Step 8. The driver who received a short-distance order in the last round will pick up the passenger through the green channel. If the driver still receives the short-distance order in this round, he can still return to the green channel of the airport to pick up passengers after completing the order. If the order received is a long-distance order, the handling method is the same as that in the second step. This goes on and on.

In the process of receiving passengers at the airport, the taxi will not return after receiving the long-distance order. If the taxi goes directly to the short-distance order, it will return to the airport and enter the green channel for the next round of passenger-carrying task.

In order to explain the above algorithm ideas, the following indicators are defined.

Then, in order to make the average profits (yuan/minute) of drivers who pick up long-distance passengers and those who pick up short-distance passengers $N$ times at the airport similar, there is a relationship between them as is shown in the following equation:

$$
\frac{a_{1}-b_{1}}{t_{1}+t_{2}}=\frac{N \times a_{2}-2 N \times b_{2}}{t_{1}+2 N \times t_{3}+N \times t_{4}} .
$$

It can be seen from the above steps that if the driver of the short distance receives a long-distance passenger in the process of transporting passengers from the airport, he will no longer come back to the airport. Therefore, $N$ can be taken to approach infinity when calculating the average profit of drivers.

Then the original relation can be changed into the two following equations:

$$
\begin{gathered}
\frac{a_{1}-b_{1}}{t_{1}+t_{2}}=\frac{N \times a_{2}-2 N \times b_{2}}{t_{1}+2 N \times t_{3}+N \times t_{4}} . \\
\frac{a_{1}-b_{1}}{t_{1}+t_{2}}=\lim _{N \rightarrow \infty} \frac{N \times a_{2}-2 N \times b_{2}}{t_{1}+2 N \times t_{3}+N \times t_{4}} .
\end{gathered}
$$

When $N$ tends to infinity, equation (35) can be approximately equal to

$$
\frac{a_{1}-b_{1}}{t_{1}+t_{2}}=\frac{a_{2}-2 b_{2}}{2 t_{3}+t_{4}}
$$

In equation (36), it is assumed that the average revenue of $N$ short-distance orders is equal to that of a long-distance order; then they are considered to be in equilibrium.

Through data collection, field research, and other methods, we can collect the data of different cities and regions $a_{1}, b_{1}, t_{1}, t_{2}$, and $t_{4}$. When $n$ approaches the limit, $a_{2}=K X, b_{2}=X b_{0}$, and $t_{3}=X / V$.

Then, substituting formula (36), we get the following formula:

$$
\frac{a_{1}-b_{1}}{t_{1}+t_{2}}=\frac{K X-2 X b_{0}}{2 X / V+t_{4}} \text {. }
$$

Solve $X$, and, compared with the actual situation of the city and Regional Airport, determine whether the solution obtained by observation is reasonable.

Taking an airport in a megacity as an example, the critical kilometers are calculated, and the rationality of the model is verified with the actual indexes given by the airport, which is shown in Table 15.

8.2. Sensitivity Analysis. As an optimization problem, sensitivity analysis of model parameters is essential:

(1) During the workdays, by investigating the average distance $d$ between an airport and the surrounding landmark buildings and business districts and 
TABLE 15: Taxi mileage and reference price from an airport to urban landmark.

\begin{tabular}{lcc}
\hline Destination & Mileage (km) & Reference fare (yuan) \\
\hline Xujiahui (Oriental Commercial Building, Ganghui Plaza, Pacific Department Store) & 48 & 180 \\
Xinzhuang & 50 & 185 \\
Lujiazui (Oriental Pearl Tower, Jinmao Tower, Zhengda Plaza) & 44 & 44 \\
Pudong Yohan Center & 37 & 165 \\
Thumb square (Lianyang Community) & 45 & 165 \\
Pentagonal field & 56 & 165 \\
Yuexing global port & 50 & 215 \\
Jing'an Temple & 48 & 185 \\
Shanghai Stadium (Shanghai Gymnasium) & 46 & 180 \\
Jinjiang paradise & 48 & 180 \\
People's Square (Urban Planning Museum) & 46 & 180 \\
Yu Garden (Town God's Temple) & 50 & 175 \\
The Bund & 39 & 185 \\
Shanghai Science and Technology Museum & 46 & 145 \\
Shanghai South Railway Station & 50 & 145 \\
Shanghai Railway Station & 50 & 185 \\
Long-distance passenger terminal & 60 \\
Hongqiao transportation hub & 47.6111 \\
Average & 230 \\
\hline
\end{tabular}

TABLe 16: The optimized calculation results from the airport to urban areas in workdays.

\begin{tabular}{lccc}
\hline $\begin{array}{l}\text { Average distance } \\
(\mathrm{km})\end{array}$ & $\begin{array}{c}\text { Percentage of short-distance orders } \\
(\%)\end{array}$ & $\begin{array}{c}\text { The average waiting time } \\
(\mathrm{min})\end{array}$ & $\begin{array}{c}\text { Threshold of long and short distance } \\
(\mathrm{km})\end{array}$ \\
\hline 47.61 & 15 & 18 & 22 \\
\hline
\end{tabular}

TABLE 17: The optimized calculation results from the airport to urban in weekends.

\begin{tabular}{lccc}
\hline $\begin{array}{l}\text { Average distance } \\
(\mathrm{km})\end{array}$ & $\begin{array}{c}\text { Percentage of short-distance orders } \\
(\%)\end{array}$ & $\begin{array}{c}\text { The average waiting time } \\
(\mathrm{min})\end{array}$ & $\begin{array}{c}\text { Threshold of long and short distance } \\
(\mathrm{km})\end{array}$ \\
\hline 57.23 & 9 & 24.1 & 29.1 \\
\hline
\end{tabular}

combining with taxi charging standards, we can get the following conclusions: $d=47.61 \mathrm{~km}$,

$a_{1}=14+(15-3) \times 2.5+(d-15) \times 3.6=178.89$,

$b_{1}=c \times d=21.08$ yuan.

According to the survey and statistics, $15 \%$ of the taxi orders from an airport are short-distance orders. In the initial conditions, taxis entering the airport for the first time have to queue for 2 hours. Therefore, the average waiting time of each taxi in the green channel is as follows:

$$
t_{4}=120 \times 15 \%=18 \mathrm{~min} .
$$

Through Baidu map software, we can get the driving distance $d$ from the airport to the urban area of the city, and the time required is $t_{2}=90 \mathrm{~min}$.

Substitute the above-mentioned $a_{1}, b_{1}, t_{1}, t_{2}$, and $t_{4}$ into formula (34).

It can be solved that $X=23.53 \mathrm{~km}$; according to the investigation, the critical distance to distinguish long distance and short distance is $22 \mathrm{~km}$, and the deviation rate between $X$ and the critical value given by the airport is calculated: $(23.53-22) \div 22=6.95 \%$ (keep two decimal places). The deviation rate between the calculated value and the actual value is less than 7\%, which belongs to the acceptable error range, so the accuracy of the model is high. The result is shown in Table 16.

(2) Meanwhile, during the weekends, calculate the above-mentioned $a_{1}, b_{1}, t_{1}, t_{2}$, and $t_{4}$ into formulas (34)-(37): we can obtain Table 17.

Comparing the parameter values of airport taxis on weekends and weekdays, it can be seen that all parameters have changed sensitively, especially the definition of the threshold of long and short distance, which fully shows that the constructed model and parameter calibration are reasonable and accurate.

\section{Conclusion}

The established decision model calculates the decisionmaking relationship, mainly around the relationship between the number of flights and the number of taxis in the pool; the final decision variable is the number of taxis in the pool $N$. In different cases, the driver only needs to compare the actual number of vehicles in the storage pool $N$ with the corresponding critical value $N_{1}$ to make a decision by comparison, which is very operable. The established mathematical model can give the corresponding opening 
number of boarding points according to the specific time period of a day and also can adjust the number of boarding points for holidays. On the premise of ensuring the evacuation of passenger flow as soon as possible, the resources can also be used reasonably. The utilization rate of boarding points is high and the model is flexible. Under the premise of ensuring the balance of long-term and long-distance order revenue, the established mathematical model is close to the actual value of long-term and long-distance critical point and has strong applicability.

The established model largely depends on the number of flights and the number of taxis in the storage pool and has insufficient discussion on other factors such as weather and has insufficient predictive power. In addition, the model established does not discuss the new maintenance cost changes due to the change of road traffic conditions, which will be discussed in the future [28].

\section{Data Availability}

The raw or processed data required to reproduce these findings cannot be shared at this time, because the data also form part of an ongoing study.

\section{Conflicts of Interest}

The authors declare that they have no conflicts of interest.

\section{Acknowledgments}

This work was supported in part by the National Key R\&D Program of China under Grant 2019YFB1600602, Shanghai Committee of Science and Technology under Grant 18DZ1206300, the National Natural Science Foundation under Grant 52072235, and the capacity building project of local colleges of Shanghai Municipal Science and Technology Commission under Grant 19030501400.

\section{References}

[1] X. Yu, S. Gao, X. Hu, and H. Park, "A Markov decision process approach to vacant taxi routing with e-hailing," Transportation Research Part B: Methodological, vol. 121, pp. 114-134, 2019.

[2] L. Yang, M.-P. Kwan, X. Pan, B. Wan, and S. Zhou, "Scalable space-time trajectory cube for path-finding: a study using big taxi trajectory data," Transportation Research Part B: Methodological, vol. 101, no. 7, pp. 1-27, 2017.

[3] G. L. Zhu, "Research on the optimization model allocation of airport taxi," Business news, vol. 186, no. 32, pp. 185-186, 2019.

[4] H. D. Yu and L. X. Zhou, "Research on airport taxi scheduling problem based on Queuing Theory," Advances in Applied Mathematics, vol. 8, no. 12, pp. 1905-1920, 2019.

[5] R. Q. Li, "Study on driver decision and management optimization of airport taxi," Journal of Statistics and Applications, vol. 9, pp. 7-18, 2020.

[6] Z. T. Zhou, "Research and analysis of taxi passenger decision making and passenger carrying optimization scheme," Science \& Technology Industry Parks, p. 254, 2019.
[7] D. P. Li, K. F. Yan, and L. J. Cheng, "Service level model of airport taxi loading area," Journal of Harbin Institute of Technology, vol. 43, no. 4, pp. 126-130, 2011.

[8] Y. X. Shi, L. Yin, and S. Y. Zhu, "Study on the setting of taxi loading area in Airport," Transportation world, vol. 000, no. 4, pp. 8-10, 2020.

[9] C. W. Yuan, Q. Q. Wu, and D. L. Wei, “Taxi market equilibrium mechanism and optimization model considering refusing to take a taxi," Journal of China highway, vol. 27, no. 6, pp. 91-97, 2014.

[10] D. W. Lee, E. J. Bass, S. D. Patek, and J. A. Boyd, "A traffic engineering model for air taxi services," Transportation Research Part E: Logistics and Transportation Review, vol. 44, no. 6, pp. 1139-1161, 2008.

[11] X. Chen, S. Wang, C. Shi, H. Wu, J. Zhao, and J. Fu, "Robust ship tracking via multi-view learning and sparse representation," Journal of Navigation, vol. 72, no. 1, pp. 176-192, 2019.

[12] Q. Luo, X. Chen, J. Yuan, X. Zang, J. Yang, and J. Chen, “Study and simulation analysis of vehicle rear-end collision model considering driver types," Journal of Advanced Transportation, vol. 2020, no. 1, 11 pages, Article ID 7878656, 2020.

[13] C. M. Fernández, V. F. Gómez, and R. M. Arnaldo, "Mathematical model for optimizing the sequence for clearing snow from the maneuvering area during winter operations," Journal of Advanced Transportation, vol. 50, no. 6, pp. 1225-1251, 2016.

[14] B. Abdelghani, M. A. Ghaleb, U. S. Suryahatmaja, and A. M. Salem, "The Airport Gate Assignment Problem: A Survey," The Scientific World Journal, pp. 9165-9172, 2014.

[15] A. R. Correia, S. C. Wirasinghe, and A. G. de Barros, "A global index for level of service evaluation at airport passenger terminals," Transportation Research Part E: Logistics and Transportation Review, vol. 44, no. 4, pp. 607-620, 2008.

[16] A. G. de Barros, A. K. Somasundaraswaran, and S. C. Wirasinghe, "Evaluation of level of service for transfer passengers at airports," Journal of Air Transport Management, vol. 13, no. 5, pp. 293-298, 2007.

[17] M. Huang, F. Mao, and Y. X. Qian, "Constrained depth reinforcement learning algorithm path mining based on taxi driver experience," Computer application research, vol. 37, no. 5, pp. 1298-1302, 2020.

[18] Y. Y. Zhang and Y. T. Tian, "Solving airport taxi queuing problem based on multi index analysis model: a case study of Chongqing Jiangbei International Airport," Statistics and application, vol. 9, no. 3, p. 10, 2020.

[19] T. Y. Zhang, Y. Y. Wu, and X. Y. Liu, "Research on decision making behavior of airport taxi drivers based on complete quadratic classifier," Journal of Xinjiang Normal University, no. 2, pp. 122-128, 2020.

[20] X. Wang, A. E. I. Brownlee, J. R. Woodward, M. Weiszer, M. Mahfouf, and J. Chen, "Aircraft taxi time prediction: feature importance and their implications," Transportation Research Part C: Emerging Technologies, vol. 124, no. 1, Article ID 102892, 2021.

[21] S. H. Kim, E. Feron, and J. P. Clarke, "Gate assignment to minimize passenger transit time and aircraft taxi time," Journal of Guidance, Control, and Dynamics, vol. 36, no. 2, pp. 467-475, 2015.

[22] T. Zhang, M. Ding, B. Wang, and Q. Chen, "Conflict-free time-based trajectory planning for aircraft taxi automation with refined taxiway modeling," Journal of Advanced Transportation, vol. 50, no. 3, pp. 326-347, 2016. 
[23] D. Ehebrecht, D. Heinrichs, and B. Lenz, "Motorcycle-taxis in sub-Saharan Africa: current knowledge, implications for the debate on "informal" transport and research needs," Journal of Transport Geography, pp. 69-76, 2018.

[24] E. A. Irvine, K. P. Shine, and M. A. Stringer, "What are the implications of climate change for trans-Atlantic aircraft routing and flight time?" Transportation Research Part D: Transport and Environment, vol. 47, pp. 44-53, 2016.

[25] M. Maciejewski, J. Bischoff, and K. Nagel, "An assignmentbased approach to efficient real-time city-scale taxi dispatching," IEEE Intelligent Systems, vol. 31, no. 1, pp. 68-77, 2016.

[26] X. Zhan, X. Qian, and S. V. Ukkusuri, "A graph-based approach to measuring the efficiency of an urban taxi service system," IEEE Transactions on Intelligent Transportation Systems, vol. 17, no. 9, pp. 2479-2489, 2016.

[27] T. Y. Li, Y. W. Chen, and D. D. Sun, "Airport taxi berth management model based on game theory," Practice and understanding of mathematics, vol. 50, no. 19, pp. 66-75, 2020.

[28] G. Porter, "Transport services and their impact on poverty and growth in rural sub-saharan africa: a review of recent research and future research needs," Transport Reviews, vol. 34, no. 1, pp. 25-45, 2014. 